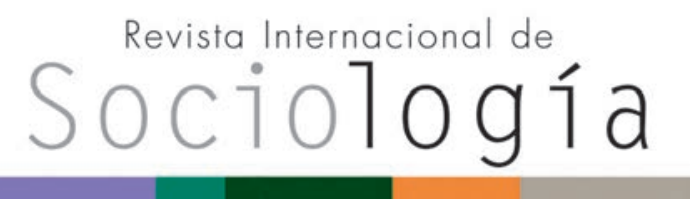

Revista Internacional de Sociología RIS vol. 76 (3), e102, julio-septiembre, 2018, ISSN-L:0034-9712 https://doi.org/10.3989/ris.2018.76.3.17.360

\section{HOMOGENEIDAD Y \\ FRAGMENTACIÓN EN LOS HÁBITOS ALIMENTARIOS DE LOS ESPAÑOLES. Una respuesta a través del análisis de los horarios, las relaciones y las normas alimentarias}

HOMOGENIZATIONS AND

FRAGMENTATION IN THE FOOD HABITS IN SPAIN. An answer based on an analysis of schedules, relationships and eating norms

\author{
Cecilia Díaz MÉndez \\ cecilia@uniovi.es \\ ORCID iD: https://orcid.org/0000-0003-3200-4922 \\ ISABEL GARCÍA ESPEJO \\ Universidad de Oviedo \\ igarcia@uniovi.es \\ ORCID iD: https://orcid.org/0000-0002-7944-3175
}

Universidad de Oviedo. Grupo de Investigación de Sociología de la Alimentación

Cómo citar este artículo / Citation: Díaz Méndez, C. e I. García Espejo. 2018. "Homogeneidad y fragmentación en los hábitos alimentarios de los españoles. Una respuesta a través del análisis de los horarios, las relaciones y las normas alimentarias". Revista Internacional de Sociología 76(3): e102. https://doi.org/10.3989/ris.2018.76.3.17.360
Copyright: ( 2018 CSIC. Este es un artículo de acceso abierto distribuido bajo los términos de la licencia de uso y distribución Creative Commons Reconocimiento 4.0 Internacional (CC BY 4.0).

Recibido: 09/03/2017. Aceptado: 01/12/17

\section{Abstract}

The objective of this article is to contribute to the debate regarding the destructuring of eating practice in contemporary societies. The data used is taken from a nationwide survey on Spanish eating habits (ENHALI, 2012). The study examines the hypothesis of the destructuring of eating habits by analysing three concrete areas. The first of these is the destructuring of timetables. Secondly, the article examines whether there exists a process of defamilisation of eating habits. The third section discusses whether eating practice can be said to be anomic. After this study it can be affirmed that the synchrony of time, the importance given to sociability and adherence to the food standard present a more homogeneous than unstructured image of the eating habits of Spanish society.

\section{KeYWORDS}

Eating practices; Family meal; Food destructuration; Sociology of food.
Comida familiar; Desestructuración alimentaria; Prácticas alimentarias; Sociología de la alimentación. 


\section{INTRODUCCIÓN}

Los análisis del cambio alimentario en Europa se han centrado en lo que se ha dado en llamar desestructuración alimentaria, un concepto que alude a la pérdida que, bajo el prefijo des, muestra cómo se van modificando los comportamientos alimentarios tradicionales de una sociedad. La desestructuración alimentaria vendría reflejada por varios rasgos referidos al cambio en los hábitos que Herpin clasificó como des-estructuración, des-socialización, desinstitucionalización, des-implantación horaria y desritualización. (Herpin 1998:505).

El proceso de individualización se manifiesta en la mayor fragmentación de la sociedad, con hábitos menos compartidos y más diversos y con el alejamiento de las pautas alimentarias tradicionales. Esto es un reflejo de la modernización de la sociedad (Gracia Arnaiz 1997; Warde 1997; Contreras 2002; Díaz Méndez y Gómez Benito 2005; Poulain 2008). Sin embargo, la falta de datos disponibles no permite resolver el debate de la estabilidad o la desestructuración de hábitos, muy especialmente en el caso español. En España las fuentes oficiales ofrecen una mirada parcial, sobre gasto alimentario y sobre el uso del tiempo, de ahí la importancia de ofrecer datos de una encuesta ad hoc (ENHALI 2012).

En este artículo se estudian, en primer lugar, los ritmos alimentarios con el objeto de analizar si se está produciendo una desimplantación horaria en nuestro país o, por el contrario, persiste una sincronía horaria y una elevada convergencia en la organización del tiempo. En segundo lugar, se aborda la existencia de un proceso de desfamiliarización de la alimentación, en relación con la pérdida del carácter relacional de la comida. Además, el artículo persigue un tercer objetivo: determinar si las normas sobre lo que se considera comer bien se han individualizado, en cuyo caso la alimentación se estaría convirtiendo en un hábito anómico, o bien siguen existiendo criterios de elección de los alimentos compartidos.

El fin último de este trabajo es aproximarse a la organización de la alimentación en España, pues aunque los datos que se van a analizar no muestran al completo la compleja realidad de la alimentación, sí presentan una visión más amplia que la habitual en este campo de estudio y, con ello, ofrecen la posibilidad de dar respuesta a la reiterada pregunta sobre la desestructuración de la alimentación que se plantea en el ámbito europeo.

\section{MARCO TEÓRICO E HIPÓTESIS}

\section{Desimplantación horaria o sincronía alimentaria}

En relación con los horarios alimentarios cabe recordar que los ritmos de la vida diaria son construcciones sociales determinadas por circunstancias históricas, sociales y culturales propias de una sociedad; de ahí que la modernidad tenga su propio orden temporal (Sorokin y Merton 1937; Zerubavel 1981). Se puede afirmar que cada sociedad crea su propio ritmo, aunque no resulte sencillo detectar qué o quién lo determina y qué o quién lo sustenta. Las actividades ligadas a la alimentación, al igual que otras actividades de la vida cotidiana, imponen sus ritmos marcando pautas de comportamiento (Grignon y Grignon 2004; Mestdag y Vandeweyer 2005). Detenerse para comer, dentro o fuera de casa; la hora a la que esto se hace y el tiempo que se le dedica, muestra los usos sociales del tiempo que se imponen a los usos de ese tiempo en otras actividades. Este uso del tiempo pone de manifiesto cómo se organiza la alimentación y qué relevancia se le da a este comportamiento en la organización del tiempo disponible. Tanto la sincronía horaria como la convergencia en la organización del tiempo disponible dan a entender que existen reglas que regulan la alimentación y que éstas son compartidas, que se respetan y que, por ello, aparecen extendidas entre los miembros de esa sociedad (Grignon y Grignon 2004; Mestdag y Vandeweyer 2005). Por el contrario, la existencia de horarios y ritmos diferenciados por grupos sociales refleja una organización diversa del tiempo disponible y de sus pautas de sincronización.

Con estas hipótesis se han realizado estudios en distintos países integrando el análisis del horario y sus cambios al análisis de la desestructuración de la alimentación. En Reino Unido se ha confirmado una alta estabilidad en la estructura general de la alimentación doméstica que se define a lo largo del siglo XX como de tres ingestas diarias: una por la mañana, una al mediodía y una comida principal al final de la tarde (Murcott 1982; Charles y Kerr 1986). Estudios más recientes indican que la norma alimentaria más generalizada entre la población británica responde a este mismo modelo, y se constata que las nuevas generaciones mantienen como comida principal y familiar la comida de la tarde, realizada en el hogar con la pareja después del trabajo (Marshall y Anderson 2002). En Francia también confirman la estabilidad de lo que denominan el modelo alimentario francés, aunque el aumento de las comidas entre horas se presenta como ejemplo de un ritmo alimentario menos regular y normativo, y se ha confirmado su expansión en la sociedad francesa (Poulain 2002).

En España los estudios antropológicos que recrean la estructura de la alimentación actual siguen hablando de dos comidas principales, la del mediodía y la de la cena (Medina 2005), aunque los horarios distan mucho de ser estables. Algunos estudios han confirmado la ampliación de los horarios en la franja del mediodía y también de la cena. Precisamente este aspecto vincula el horario alimentario con la persistencia de la comida familiar, pues para estos autores la ampliación horaria se debe a la diversidad 
de situaciones escolares y laborales que impiden a los miembros de la familia comer juntos (Contreras y Gracia 2004). Otros estudios confirman una importante estabilidad horaria en España en comparación con otros países (Díaz Méndez y García Espejo 2014; Díaz Méndez y Callejo 2014).

En definitiva, los estudios sobre los horarios alimentarios muestran tanto cambios como estabilidades, y se entiende que todo aquello que rompe los ritmos y los usos tradicionales del tiempo es un rasgo de la fragmentación temporal de la alimentación propia de la modernidad social.

\section{Desfamiliarización de los hábitos alimentarios}

El hogar desempeña un papel central en el proceso de cambio alimentario, pues es el espacio en el que se desarrolla la cultura alimentaria de una sociedad; ya no sólo porque tradicionalmente se hayan resuelto en su seno las necesidades alimentarias de los individuos, sino porque es donde se trata culturalmente la comida a través de su preparación y su consumo colectivo. Además, como agentes primarios de socialización, la familia y el hogar transmiten las pautas culturales necesarias para la reproducción de estos hábitos, dándoles continuidad.

Por todo ello, la pérdida de la comida familiar se ve con preocupación. La fragmentación en las elecciones y el abandono de los referentes alimentarios tradicionales podrían conducir a los individuos hacia conductas inapropiadas, y distorsionar la continuidad cultural de unos hábitos alimentarios que han dado muestras de ser adecuados, como sucede con las dietas mediterráneas, que se asocian a la alta esperanza de vida y a la buena salud de la población de los países del sur de Europa. Pero, más allá de las cuestiones ligadas a la salud, la pérdida de la comida familiar se ve como un alejamiento del grupo familiar y del origen social del individuo, así como el abandono del carácter convivencial y colectivo que definió a la alimentación en el pasado. La pérdida del referente familiar en la alimentación mostraría, en definitiva, el peso de nuevos grupos de referencia para decidir qué y cómo comer y, con ello, el camino hacia un proceso de homogeneización alimentaria y pérdida de identidad cultural (Murcott 1982; Herpin 1998).

Para confirmar el abandono de los hábitos alimentarios ligados al grupo familiar o rebatir las tendencias a la homogeneidad alimentaria, una parte de los trabajos empíricos sobre este tema estudian períodos de tiempo amplios y otros comparan varios países. Diversos autores confirman que el tiempo dedicado a comer en el hogar no ha disminuido significativamente a lo largo de los años, aunque también constatan que la organización temporal de la alimentación difiere entre países, conservando así especificidades nacionales (Warde et al. 2007; Cheng et al. 2007; Díaz Méndez y García Espejo 2014).
Otros trabajos, basados en estudios longitudinales en una misma sociedad, sí muestran una reducción del tiempo dedicado a comer en grupo, aunque en ningún caso un drástico abandono de esta práctica (Mestdag y Glorieux 2009). Por otra parte, también se observa continuidad en las creencias y los valores asociados a la comida familiar y, al estudiar a jóvenes y adolescentes, se confirma que la comida doméstica se asocia a comida sana y apropiada, tanto en diferentes generaciones como entre la población urbana y rural (Lupton 1996; Gallegos et al. 2011). Además, la comida es, con frecuencia, una manifestación de la unidad del grupo familiar (Kemmer, Anderson y Marshal 1998; Sobal, Bove y Rauschenbach 2002).

Los estudios que analizan la comida extradoméstica llegan a conclusiones menos contundentes respecto a la conservación de la comida. Estas investigaciones confirman el crecimiento de la alimentación extradoméstica en todas las sociedades: aumenta tanto el número de personas que comen fuera del hogar como en el tiempo que dedican a ello, aunque este aumento no siempre se corresponde con el descenso de la comida familiar (Warde et al. 2007; Cheng et al. 2007; Davidson y Gauthier 2010; MartínLagos 2011).

En general, se podría decir que los estudios empíricos no indican una drástica desaparición de la comida familiar, ni tampoco una orientación contundente hacia pautas alimentarias más homogéneas y externas al hogar. Más bien se aprecia que la estandarización de algunos hábitos convive con pautas alimentarias de origen nacional, sobre las que se asientan las tendencias más generales de cambio.

\section{Pérdida e individualización de las normas alimentarias. ¿Hay anomia alimentaria?}

La posibilidad de una anomia aplicada a la alimentación fue planteada por Claude Fischler en el año 1979, cuando muestra la falta de normas de la alimentación contemporánea. En este trabajo desarrolla el dilema entre gastronomía y gastro-anomía. Según este autor, en las sociedades contemporáneas se ha modificado la función reguladora del sistema culinario del comensal. Esto da lugar a un debilitamiento de las normas que dificultan la elección de los alimentos (anomía gastronómica). Cada vez con más frecuencia, los dispositivos de regulación social pierden eficacia y aparecen criterios diversos y, a veces, contradictorios. Es lo que el autor denomina cacofonía alimentaria (Fischler, 1979: 206).

Esta anomia alimentaria se produce por la pérdida de peso de la clase social para explicar los comportamientos alimentarios. No es algo apoyado por todos los autores, pues hay detractores y partidarios que, con sus trabajos empíricos, han ido mostrando el mayor o menor peso de la clase social para explicar el cambio alimentario. 
En Francia, en los años ochenta, Grignon y Grignon mostraron las diferencias entre las comidas de ricos y pobres (Grignon y Grignon 1980; 1981). Los trabajos de Bourdieu son también una referencia para quienes postulan la pervivencia de las clases sociales. Bourdieu, analizando la estructura de consumo y el gasto en Francia, establece una diferenciación en los consumos alimentarios de los distintos grupos ocupacionales, constatando el efecto de la clase sobre la alimentación (Bourdieu 1998: 180). Bourdieu confirma la hipótesis de las desigualdades de clase en el consumo alimentario y confirma que las normas de clase siguen vigentes en los noventa. También Lambert, basándose en Bourdieu y Elias, plantea la existencia de dos modelos alimentarios de clase: tradicional (en decadencia) y moderno (en expansión), a través de los cuales describe la adscripción de los franceses a estos modelos alimentarios en función de su clase social (Lambert 1987). Todos ellos explican, desde diferentes perspectivas y con el foco de atención puesto en las clases sociales, cómo se construyen las normas alimentarias.

Warde en Reino Unido confirma las diferencias de clase en los comportamientos alimentarios y la diferencia entre la clase obrera y la clase media (Warde 1997: 118). Pero también constata un fraccionamiento de las clases medias y la aparición de pautas de consumo, como las elecciones por salud o belleza, que se da por igual en todos los grupos sociales (Warde 1997). Las normas comienzan a diversificarse y a no aparecer de manera tan clara vinculadas al grupo social de pertenencia. No obstante, otros autores también han confirmado que el capital cultural sigue marcando pautas de diferenciación en la sociedad británica (Atkinson y Deeming 2015).

En España también se ha estudiado la relación entre alimentación y clase social y tanto González Turmo (1995) como Gracia Arnaiz (2003) muestran que persisten las diferencias de clase en los hábitos alimentarios, y que aún se puede hablar de comidas de ricos y las comidas de pobres.

El planteamiento teórico más elaborado ha sido desarrollado por Warde, quien sostiene que siguen presentes las normas grupales ligadas al origen social, pero considera que es excesivamente simple establecer una dualidad entre lo grupal y lo individual. Para Warde es más apropiado hablar de fuerzas sociales contrapuestas que actúan de manera simultánea. Estas fuerzas actúan sobre los comportamientos alimentarios en una doble dirección: la individualización y la integración comunitaria, por un lado, y la estilización y la informalización por otro. El modelo de Warde muestra cuatro pautas de diferenciación en la elección de la comida, basadas en la confluencia diversa de las cuatro fuerzas sociales antes planteadas (Warde 1997: 42). Estas fuerzas dan lugar a una tipología de comportamientos alimentarios que rompen con la dualidad, pues no se posiciona ni en la pluralidad de elecciones que plantean los partidarios de la individualización, ni en la explicación basada en las clases sociales de los partidarios de la reproducción social.

A partir de este marco teórico, en este artículo se van a considerar un conjunto de conductas alimentarias que describen los hábitos alimentarios de los españoles, siguiendo el planteamiento de Herpin sobre la desestructuración alimentaria: horarios, relaciones y normas. En primer lugar, se analizan las franjas horarias ligadas a la alimentación cotidiana para averiguar si estamos ante horarios fijos y compartidos por toda la sociedad o, por el contrario, ante horarios flexibles y orientados a adaptarse a los ritmos y dinámicas de los individuos. En segundo lugar, se estudian las relaciones ligadas a la alimentación para averiguar si estamos ante unas conductas alimentarias individuales o si el grupo familiar coincide para comer. En tercer lugar, se exploran las normas alimentarias para comprobar si se detectan reglas de conducta diferenciadas según los individuos o si, antes bien, existen pautas de elección de la comida compartidas que trascienden las diferencias sociodemográficas. Todo ello nos permite averiguar si en España la alimentación es una actividad desestructurada o, por el contrario, un hábito homogéneo y compartido por la población.

\section{FUENTE DE DATOS Y ANÁLISIS ESTADÍSTICO}

Para dar respuesta a las preguntas e hipótesis que se plantean en este artículo, se ha utilizado como fuente de datos la "Encuesta de Hábitos Alimentarios" (ENHALI 2012) de ámbito nacional y realizada a 1504 personas mayores de 18 años. Se ha trabajado con un error muestral del $+/-2,5 \%$ y un nivel de confianza del $+/-2$ sigmas, o del $95,5 \%$. Se trata de una encuesta telefónica realizada por el sistema CATI (Computer Assisted Telephone Interviewing) siguiendo un método de muestreo polietápico por el que se selecciona, en primer lugar, los municipios españoles (dentro de cada comunidad autónoma) $y$, en segundo lugar, a los individuos por cuotas de edad y sexo. Estas cuotas responden a las de la población española. Es sabido que en las encuestas telefónicas el nivel de rechazo por parte de los potenciales encuestados suele ser más alto que en las encuestas presenciales. Para paliar este problema, en los casos de ausencia de respuesta se ha sustituido al encuestado por un perfil idéntico siguiendo las cuotas del estudio: edad, sexo y municipios.

En la encuesta se pregunta, en primer lugar, por los aspectos estructurales de la alimentación, aquellos que constituyen el soporte de los hábitos. Así, se exploran los horarios y los lugares en los que se come y los aspectos de sociabilidad ligados a las comidas. Seguidamente se aborda la preparación de la 
comida y las decisiones sobre la dieta. En la encuesta también se indaga por la situación alimentaria actual, el grado de preocupación de la población ante los riesgos alimentarios y la confianza en las instituciones. Finalmente, se abordan las actitudes acerca de la comida y los cambios alimentarios.

Conviene señalar que no es posible responder a las cuestiones que exploran globalmente los hábitos alimentarios de los españoles con las fuentes estadísticas oficiales existentes. En primer lugar, la Encuesta de Presupuestos Familiares del Instituto Nacional de Estadística ignora los aspectos relativos a los hábitos, centrándose en el gasto alimentario. En segundo lugar, el Panel de Consumo Alimentario del Ministerio de Agricultura, Alimentación y Medio Ambiente no ofrece las bases de datos para su explotación. En tercer lugar, la Encuesta de Empleo del Tiempo del Instituto Nacional de Estadística muestra rutinas, pero carece de datos referidos a las actitudes. En definitiva, solo una encuesta ad hoc permite responder a aspectos relativos a los hábitos alimentarios e integrar, en un misma fuente, los diversos aspectos planteados por separado en las fuentes oficiales existentes en España.

Para el análisis de los resultados obtenidos se ha aplicado la técnica de regresión logística. En concreto, la regresión logística multinomial para analizar los horarios de las comidas y las cenas de los españoles. Para abordar los aspectos relacionales y de sociabilidad ligados a las comidas se ha aplicado la técnica de regresión logística binaria. Por último, para determinar la asociación entre los criterios que intervienen en la elección de los alimentos y las variables sociodemográficas se han realizado pruebas de significación para preguntas múltiples.

\section{¿HAY DESIMPLANTACIÓN HORARIA O SINCRONÍA EN LOS HÁBITOS ALIMENTARIOS?}

En términos generales, los españoles organizan su alimentación cotidiana comiendo tres veces al día: el desayuno (97\% de la población), la comida (99\%) y la cena (98\%). Una buena parte de ellos también realiza otras dos ingestas: un tentempié de media mañana (36\%) y una merienda (44\%). Estos datos se refieren a la realización de estas comidas el día anterior a la entrevista, independientemente de donde se hayan consumido, en casa o fuera de casa.

La mayoría de los encuestados afirma comer entre tres y cuatro veces al día $(42,6 \%$ y $33,4 \%$ respectivamente) con una media de 3,7 ingestas diarias, una cifra muy parecida a la que presentan los franceses (OCHA 1993; Lhuissier et al. 2013). En el caso de las tres comidas se refieren al desayuno, la comida y la cena y, cuando se señalan cuatro ingestas, se añade la merienda. Cerca del $21 \%$ realiza cinco comidas diarias. Del resto, sólo un $2 \%$ come dos veces al día y un $1 \%$ más de cinco veces. Estos datos coinciden bastante con los obtenidos en otros estudios de años anteriores (Contreras y Gracia 2004; Gracia 2008), lo cual sugiere que todavía existe una gran estabilidad en los hábitos alimentarios de los españoles.

La mayoría come entre las 14 y las 15 horas, aunque uno de cada cuatro españoles come después de las 3 de la tarde. La cena tiene un horario más amplio, con dos picos relevantes. La mayoría de la población española cena después de las nueve de la noche (85\%): un nutrido grupo lo hace entre las $21 \mathrm{y}$ las $21,30(41 \%)$, aunque casi dos de cada tres lo hace después de esa hora (Figura 1).

Figura 1.

Horarios de comidas y cenas (porcentajes)

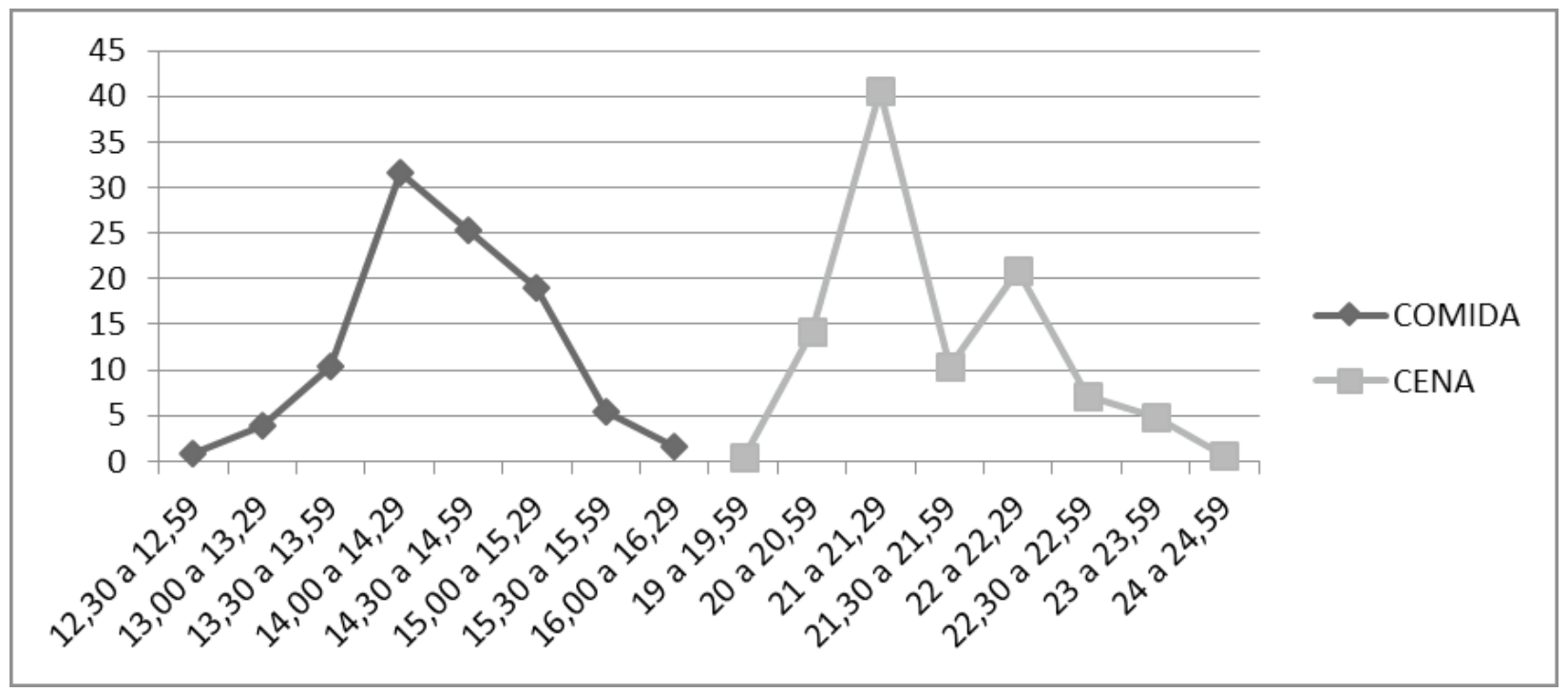

Fuente: ENHALI-2012. 
La comida principal con mayor amplitud horaria es el desayuno, que va desde las 7 a las 11 de la mañana. La mayoría de la población desayuna entre las 8 y las 10 de la mañana (61\%); un $28 \%$ lo hace entre las 8 y las 9 y un $33 \%$ entre las 9 y las 10 . Se trata de una ingesta mayoritaria y realizada en el hogar, aunque un $6 \%$ lo hace fuera de casa y solo un $3 \%$ de la población no desayuna. Aunque se ha comentado en otros estudios que el desayuno es una ingesta menos estructurada (Serra Majem et al. 2003), se podría decir de modo más preciso que estamos ante una ingesta con un horario más amplio, lo que no es tanto un rasgo de desestructuración como de dispersión horaria. La concentración de personas haciendo lo mismo a la misma hora es mayor en la comida, pues se produce en un intervalo de una hora, mientras que esta concentración se da en el desayuno en un período de dos horas.

Si nos referimos al número de veces que se come al día en casa y fuera de casa, la media española se sitúa en 2,8 ingestas para la comida doméstica y 0,9 en el caso de la comida extradoméstica. Estos datos son muy parecidos a los obtenidos a partir de la Encuesta de Empleo del Tiempo (Instituto Nacional de Estadística 2010; Díaz Méndez y García Espejo 2014) y contrastan con los de otros países europeos, como el Reino Unido, donde el número medio de comidas diarias dentro de casa es sensiblemente menor $(1,2)$, mientras que es mayor la media de comidas extradomésticas $(1,5)$ (Multinational Time Use Study 2011; Díaz Méndez y García Espejo 2014). También la duración de las comidas es superior en España que en el Reino Unido (Díaz Méndez y Callejo 2014).

Para profundizar en el conocimiento de estas ingestas es necesario conocer el perfil de quien las realiza, pues la mera descripción de frecuencias no permite ver la asociación real que existe entre las variables estudiadas. Es por ello que se efectúa un análisis más exhaustivo, recurriendo a técnicas multivariantes que permiten determinar cuáles son los factores más relevantes a la hora de explicar la organización alimentaria de la población española en términos de horario.

Se ha recurrido a distintos modelos de regresión logística referidos a las ingestas principales: la comida del mediodía y la cena. Las variables dependientes son los grupos denominados tempraneros, estándar y tardíos. Con este fin se han agrupado los entrevistados en función del horario en estas tres categorías (Cuadro 3): En la comida denominamos tempraneros a quienes comen antes de las 14 horas (14\%), estándar a los que lo hacen entre las 14 y las 15 horas (59\%) y tardíos a quienes comen después de las tres de la tarde (27\%). En la cena los tempraneros serían aquellos que cenan antes de las 9 de la noche (15\%), los estándar entre las 9 y las $10(51 \%)$ y los tardíos quienes retrasan la cena a horas posteriores a las 10 de la noche (34\%).
En la comida y la cena se han aplicado regresiones logísticas multinomiales, en las que el valor de comparación "0" serán los casos que hemos denominado estándar, que corresponden a los individuos que comen y cenan en la franja horaria más frecuente. A ellos se opondrá, sucesivamente, el grupo de los que realizan las comidas y cenas más temprano (tempraneros) y los que las hacen más tarde (tardíos). Con estos modelos no se pretende la cuantificación de probabilidades, sino el análisis de la correlación o asociación de las variables que se han considerado relevantes. La explicación de los resultados obtenidos se centra en aquellos factores que se asocian positivamente con las distintas situaciones horarias. Las variables independientes incluidas en los modelos son el sexo, la edad, el número de habitantes del lugar de residencia, el tipo de hogar, el nivel de estudios y las situaciones de la población respecto a la actividad laboral.

Si nos referimos al horario de las comidas del mediodía, el factor que determina que se coma más temprano o más tarde es la situación laboral (Cuadro 1). Así, los que comen más pronto son los jubilados, mientras que los que realizan esa actividad más tardíamente son especialmente los que trabajan y también los parados y los estudiantes. No hay ningún otro factor que sea significativo y que se asocie positivamente con las diferentes pautas de organización de la comida del mediodía. Disminuye la probabilidad de comer más tarde vivir en poblaciones de 2000 a 10.000 habitantes o de 10.001 a 100.000 y tener un nivel de estudios de EGB/ESO, aunque el nivel de significación de esta última categoría es muy bajo.

En relación con los horarios de las cenas, no hay ninguna característica sociodemográfica que explique la adopción de un horario temprano para cenar. Sin embargo, los tardíos cuentan con un perfil más claro: son los hombres, los más jóvenes (aquellos con edades comprendidas entre 18 y 40 años) y los que se encuentran parados, trabajan o estudian. Con un nivel de significación menor, presentan también una asociación positiva con cenar más tarde los parados o estudiantes, los que tienen un nivel educativo de EGB/ESO y los que viven en poblaciones pequeñas, de 2001 a 10.000 habitantes.

En resumen, los datos sobre los horarios alimentarios de los españoles están mostrando una importante homogeneidad en los comportamientos que se pone de manifiesto, en especial, en dos aspectos: por un lado, en la regularidad horaria; por otro lado, en que las comidas se realizan mayoritariamente en el hogar. Las comidas secundarias están menos implantadas y tienen mayores irregularidades, por lo que cabe suponer que la estabilidad alimentaria se sustenta en tres comidas principales diarias: el desayuno, la comida y la cena. 
Cuadro 1.

Regresión multinomial. Horarios de comidas y cenas

\begin{tabular}{|c|c|c|c|c|}
\hline & $\begin{array}{l}\text { Tempraneros } \\
\text { Comida }\end{array}$ & $\begin{array}{l}\text { Tardíos } \\
\text { Comida }\end{array}$ & $\begin{array}{l}\text { Tempraneros } \\
\text { Cena }\end{array}$ & $\begin{array}{c}\text { Tardíos } \\
\text { Cena }\end{array}$ \\
\hline Sexo (Cat. residual: mujer) & $-0,079$ & $-0,039$ & 0,171 & $0,367^{* * *}$ \\
\hline \multicolumn{5}{|l|}{ Edad (Cat. residual: más de 75 años) } \\
\hline $18-30$ & $-0,188$ & 0,084 & $-0,788$ & $0,964^{* * *}$ \\
\hline $31-40$ & 0,021 & $-0,028$ & $-0,218$ & $0,793^{* *}$ \\
\hline $41-55$ & $-0,323$ & 0,129 & $-0,134$ & $-0,028$ \\
\hline $56-65$ & $-0,156$ & 0,153 & $-0,206$ & 0,436 \\
\hline $66-75$ & $-0,231$ & $-0,279$ & 0,027 & $-0,142$ \\
\hline \multicolumn{5}{|c|}{$\begin{array}{l}\text { Tamaño de hábitat (Cat. residual: más de } 500.000 \\
\text { habitantes) }\end{array}$} \\
\hline Hasta 2000 habitantes & $-0,534$ & $-0,428$ & $-0,170$ & $-0,400$ \\
\hline De 2001 a 10.000 habitantes & $-0,332$ & $-0,448^{*}$ & $-0,028$ & $0,435^{*}$ \\
\hline De 10.001 a 100.000 habitantes & 0,242 & $-0,678^{* * *}$ & 0,046 & $-0,007$ \\
\hline De 100.001 to 500.000 habitantes & 0,020 & 0,025 & $-0,261$ & 0,235 \\
\hline \multicolumn{5}{|l|}{ Tipo de hogar (Cat. residual: solo) } \\
\hline Pareja/cónyuge & 0,109 & $-0,156$ & $-0,246$ & $-0,283$ \\
\hline Pareja/cónyuge e hijos & 0,163 & 0,107 & $-0,443$ & $-0,175$ \\
\hline Hijos & $-0,338$ & $-0,084$ & $-0,338$ & $-0,115$ \\
\hline Padres & $-0,152$ & 0,116 & 0,017 & 0,030 \\
\hline Madre o Padre & $-0,088$ & $-0,159$ & 0,563 & 0,090 \\
\hline Amigos & 0,679 & $-0,065$ & $-1,233^{*}$ & $-0,084$ \\
\hline Otras situaciones & $0,856^{* *}$ & 0,276 & 0,030 & $-0,092$ \\
\hline \multicolumn{5}{|c|}{$\begin{array}{l}\text { Nivel de estudios (Cat. residual: universitarios } \\
\text { superiores) }\end{array}$} \\
\hline Menos que primarios & 0,284 & $-0,018$ & $-0,575$ & 0,147 \\
\hline EGB, ESO & 0,183 & $-0,368^{*}$ & $-0,329$ & $0,334^{*}$ \\
\hline Bachiller, BUP, COU & $-0,235$ & $-0,180$ & $-0,412$ & 0,166 \\
\hline FP & 0,217 & $-0,245$ & $-0,354$ & 0,019 \\
\hline Universitarios medios & 0,197 & $-0,032$ & $-0,572^{*}$ & 0,132 \\
\hline \multicolumn{5}{|c|}{ Situación laboral (Cat. residual: ama de casa) } \\
\hline Trabaja & 0,271 & $1,063^{* * *}$ & $-0,588^{* *}$ & 0,300 \\
\hline Parado & $-0,156$ & $0,641^{* *}$ & $-0,162$ & $0,478^{*}$ \\
\hline Estudiante & $-0,456$ & $0,983^{* * *}$ & $-1,125$ & $0,590^{*}$ \\
\hline Jubilado & $0,528^{* *}$ & 0,127 & $-0,221$ & $-0,063$ \\
\hline N 1504 & & & & \\
\hline
\end{tabular}

Tanto los horarios como los lugares están mostrando una sociedad bastante homogénea. Los aspectos que parecen introducir variaciones tienen que ver con los ritmos laborales. Los individuos insertos en el mercado de trabajo y los adultos que estudian son los responsables de la ampliación de los horarios de las comidas. La estabilidad alimentaria, sin embargo, guarda relación con el hecho de comer y cenar en el hogar. Cabe plantear si comer en familia es lo que estimula y refuerza la regularidad de este comportamiento o si se trata solo de un hábito que se ve favorecido por la presencia de una persona que cocina en el hogar. El hecho de que los horarios de las cenas sean amplios anima a pensar que, aunque se coma en casa, no se come necesariamente con la familia; más bien se puede sospechar que algún familiar se hace cargo de las actividades alimentarias, adaptándose a las irregularidades que el estudio, el trabajo o el ocio introducen en el horario de la cena.

Los factores que imprimen pautas horarias en la alimentación no son los mismos en las comidas del mediodía que en las cenas, ni tampoco en las ingestas principales y las secundarias; por ello, no se pue- 
den comprender apelando a los mismos factores. No obstante, lo que sí parece unificar la organización de la alimentación cotidiana es el hogar como espacio principal (y casi exclusivo) de consumo, pues es en él dónde se resuelven mayoritariamente las necesidades alimentarias cotidianas.

Es evidente que esta información nos aproximaría más al concepto de homogeneización si se pudiera contar con el tipo de ingestas realizadas por cada uno de los miembros del hogar, pues siempre cabe la posibilidad de que las personas coman juntas y en casa, pero con diferentes dietas, lo que estaría reflejando una heterogeneidad interna que no muestran los datos más generales. No obstante, no es posible contar con esta información, ni en esta ni en otras encuestas, dado que, al no contar con datos desagregados por hogar, no es posible saber qué come cada cual dentro de un mismo hogar.

Avanzando en la explicación, no obstante, hay una cuestión que analizar más a fondo con el objetivo de comprender el uso generalizado del hogar como lugar de consumo alimentario: las relaciones sociales que se establecen en su seno. Para clarificar la dimensión social del hecho alimentario es preciso explorar con quién se come. Se trataría de ver en qué medida los aspectos sociales ligados al hogar son motivadores de este comportamiento, pero también la forma en que resuelven su alimentación las personas que viven solas, cuyo elemento motivador para comer en casa no puede ser, evidentemente, la compañía.

\section{¿SE HA PERDIDO LA COMIDA FAMILIAR?}

La pérdida de la comida familiar es un asunto que ha suscitado gran número de trabajos académicos sobre el cambio alimentario, al considerar que su deterioro es un reflejo de la individualización alimentaria y un efecto no deseado de los cambios en la alimentación actual. Las comidas en soledad irían en detrimento de los aspectos sociales y relacionales de la alimentación y contra la vinculación familiar del hecho alimentario. Al margen de la valoración inherente a algunos de estos estudios, los analistas que han comparado la alimentación de varios países no han corroborado que la comida en familia haya dado paso a la comida en solitario frente al televisor (Warde et al. 2007). Por otra parte, las encuestas europeas muestran que la sociabilidad es uno de los atributos considerados más relevantes a la hora de definir una buena alimentación (Eurobarometer 2010). Se podría decir que comer en soledad no es una situación atractiva para el comensal, pero los cambios demográficos indican que ha aumentado de manera significativa el número de hogares unipersonales, por lo que cabe pensar en los aspectos relacionales de la comida por el hecho de vivir solo.
Los datos sobre los españoles no muestran un proceso de individualización alimentaria si tenemos en cuenta, como se ha visto, que la gente come en su casa y en un horario relativamente concordante. Además, la importancia de comer en compañía se pone de manifiesto si comparamos los datos españoles con los procedentes de otros contextos. Así, mientras que en España más del $80 \%$ de la población come en compañía de otras personas, tanto en la comida como en la cena, en países como Dinamarca, Noruega y Suecia el porcentaje de la comida en compañía gira en torno al $70 \%$ y en Finlandia supera ligeramente el 65\% (Holm, Kjaernes y Laurdsen 2014).

No obstante, la comida familiar no tiene el mismo peso en todas las ingestas diarias y muestra un perfil de convivencia diferente según el momento del día (Cuadro 2). Las comidas con mayor componente relacional del día son las centrales, la comida y la cena, y se realizan principalmente con la familia; pero también el tentempié de la mañana se hace con el grupo familiar. Las comidas más solitarias son la merienda y el desayuno, pues al menos uno de cada tres españoles realiza en soledad la esta ingesta (32\% y $38 \%$ respectivamente).

En general, las comidas secundarias las realiza en soledad la gente que vive sola. Aunque resulta una obviedad no lo es tanto, pues un $27 \%$ de las personas que viven solas realizan la comida del mediodía con familiares o amigos y en torno al $25 \%$ también cenan acompañadas. En más del $80 \%$ del resto de los hogares se realizan la comida y la cena con familiares (Cuadro 3 ). Todo parece indicar que la sociabilidad alimentaria es un asunto que define el hábito de la mayoría de los comensales, aunque residir solo imprime una lógica restricción de este comportamiento.

Estos resultados se complementan con un análisis multivariable de regresión logística en el que la variable dependiente asume los valores de 1 (comer en compañía) y 0 (comer solo). Las variables independientes son la edad, el tamaño del hábitat, el tipo de familia, los estudios, la situación laboral y los horarios de comidas (tempraneros, estándar y tardíos). También se ha considerado una variable independiente que combina el sexo y la responsabilidad en la preparación de la comida con los valores de 1 (hombre con responsabilidad), 2 (mujer con responsabilidad), 3 (hombre sin responsabilidad) y 4 (mujer sin responsabilidad). Se han diseñado modelos tanto para la comida de mediodía como para la cena (Cuadro 4).

Los resultados obtenidos refuerzan la importancia del familismo y la sociabilidad en España. Así, como se puede ver en los resultados obtenidos, la variable fundamental y más significativa es el tipo de familia: comen y cenan en compañía los que viven en compañía de otras personas, especialmente en los 
Cuadro 2.

Con quién se realizó cada tipo de comida

\begin{tabular}{|lccccc|}
\hline & Desayuno & Tentempié & Comida & Merienda & Cena \\
\hline Solo & 38,4 & 35,0 & 17,8 & 31,9 & 18,5 \\
Familia & 57,6 & 41,4 & 77,6 & 58,4 & 78,4 \\
Amigos & 1,4 & 6,3 & 1,5 & 3,7 & 2.1 \\
Compañeros de trabajo & 2,3 & 14,8 & 2,7 & 4,4 & 0,7 \\
Otros & 0,4 & 2,5 & 0,3 & 1,6 & 0,3 \\
Total & 100,0 & 100,0 & 100,0 & 100,0 & 100,0 \\
\hline
\end{tabular}

Fuente: ENHALI-2012.

Cuadro 3.

Con quién se realizó la comida y la cena según el tipo de convivencia

\begin{tabular}{|lccccc|}
\hline & Solo & Con pareja & Pareja e hijos & Padres y hermanos & Otras \\
\hline Comida & & & & & \\
Solo & 67,9 & 7,4 & 10,1 & 10,8 & 14,4 \\
Familia & 25,5 & 88,4 & 86,7 & 82,7 & 78,4 \\
Amigos & 1,0 & 1,1 & 0,4 & 4,9 & 3,2 \\
Compañeros de & 4,6 & 2,9 & 2,7 & 1,6 & 2,4 \\
trabajo & 1,0 & 0,2 & -- & -- & 1,6 \\
Otros & 100,0 & 100,0 & 100,0 & 100,0 & 100,0 \\
Total & & & & & \\
\hline Cena & 75,5 & 7,9 & 8,1 & 14,3 & 16,1 \\
Solo & 21,3 & 89,8 & 90,6 & 77,8 & 77,4 \\
Familia & 1,6 & 1,1 & 0,4 & 7,9 & 4,0 \\
Amigos & 0,5 & 0,9 & 0,9 & -- & 0,8 \\
Compañeros de & 1,1 & 0,2 & -- & -- & 1,6 \\
trabajo & 100,0 & 100,0 & 100,0 & 100,0 & 100,0 \\
Otros & & & \\
Total & & & & & \\
\hline
\end{tabular}

Fuente: ENHALI-2012.

casos en que se vive con la pareja/conyuge y con hijos, es decir, se vive en compañía y no se come separadamente. Cabe destacar que en España la mayoría de los hogares no son unipersonales y las personas que viven solas constituyen un porcentaje reducido, aunque en aumento; en concreto, uno de cada cuatro españoles vive solo (Instituto Nacional de Estadística 2011).

Del resto de variables, la única que resulta significativa es la responsabilidad en la preparación de los alimentos. Ni la edad, ni el tamaño del hábitat (rural o urbano), ni los estudios, ni la situación laboral, ni los horarios afectan a la probabilidad de comer en compañía. Tanto en los hombres como en las mujeres, quienes tienen más probabilidad de comer con otras personas son aquellas que no tienen ninguna responsabilidad en la preparación de los alimentos; es otra persona, pues, quien tiene que prepararles las comida. Por el contrario, ser responsable de la preparación de la comida se asocia negativamente con la realización de esta práctica en compañía. En esta categoría estarían, obviamente, aquellas personas que viven solas y que asumen en mayor medida estas tareas.

En definitiva, los datos de la encuesta ENHALI-2012 muestran una permanencia casi universal del hábito de realización de comidas en casa, en familia y en horarios relativamente concordantes entre sus miembros. La comida en el hogar y en compañía vertebra las ingestas principales y muestra la preferencia de los españoles por los aspectos relacionales de la alimentación, así como por el hogar como lugar de consumo prioritario. La comida en soledad, no obstante, está presente también de modo especial en algunos colectivos y es muy relevante en las ingestas secundarias y en el desayuno. 


\section{Cuadro 4.}

Regresión logística. Comer y cenar en compañía

\begin{tabular}{|c|c|c|}
\hline \multicolumn{3}{|l|}{ Edad (Cat. residual: más de 75 años) } \\
\hline $18-30$ & 0,229 & 0,407 \\
\hline $31-40$ & $-0,133$ & 0,421 \\
\hline $41-55$ & 0,442 & 0,623 \\
\hline $56-65$ & 0,069 & 0,210 \\
\hline $66-75$ & 0,185 & 0,335 \\
\hline \multicolumn{3}{|c|}{ Tamaño de hábitat (Cat. residual: más de 500.000 habitantes.) } \\
\hline Hasta 2000 habitantes & 0,114 & $-0,672$ \\
\hline De 2.001 a 10.000 habitantes & 0,569 & $-0,290$ \\
\hline De 10.001 a 100.000 habitantes & 0,413 & 0,059 \\
\hline De 1000.001 a 500,000 habitantes & 0,387 & 0,034 \\
\hline \multicolumn{3}{|l|}{ Tipo de hogar (Cat. residual: solo) } \\
\hline Pareja/cónyuge & $3,072^{\star \star *}$ & $3,400^{* * *}$ \\
\hline Pareja/cónyuge e hijos & $2,815^{\star \star *}$ & $3,392^{* * *}$ \\
\hline Hijos & $2,078^{* * *}$ & $2,588^{* * *}$ \\
\hline Padres & $1,957^{* * *}$ & $2,062^{* * *}$ \\
\hline Padre o madre & $1,959^{* * *}$ & $2,225^{* * *}$ \\
\hline Amigos & $2,671^{* * *}$ & $2,723^{* * *}$ \\
\hline Otras situaciones & $2,559^{* * *}$ & $3,132^{* * *}$ \\
\hline \multicolumn{3}{|c|}{ Nivel de estudios (Cat. residual: universitarios superiores) } \\
\hline Menos que primarios & $-0,424$ & 0,204 \\
\hline EGB, ESO & $-0,131$ & 0,109 \\
\hline Bachiller, BUP, COU & $-0,184$ & 0,024 \\
\hline FP & 0,059 & 0,088 \\
\hline Universitarios medios & $-0,133$ & 0,196 \\
\hline \multicolumn{3}{|c|}{ Situación laboral (Cat. residual: ama de casa) } \\
\hline Trabaja & $-0,636$ & $-0,360$ \\
\hline Parado & $-0,155$ & 0,159 \\
\hline Estudiante & $-0,182$ & $-0,003$ \\
\hline Jubilado & $-0,171$ & $-0,176$ \\
\hline \multicolumn{3}{|c|}{$\begin{array}{l}\text { Responsabilidad de la preparación de alimentos y sexo (Cat. residual: mujer } \\
\text { sin responsabilidad }\end{array}$} \\
\hline Hombre con responsabilidad & $-1,061^{* * *}$ & $-0,950^{\star * *}$ \\
\hline Mujer con responsabilidad & $-0,984^{* * *}$ & $-1,001^{* * *}$ \\
\hline Hombre sin responsabilidad & 0,166 & $-0,085$ \\
\hline \multicolumn{3}{|c|}{ Horario de las comidas (Cat. residual: Tempraneros) } \\
\hline Estándar & 0,377 & 0,402 \\
\hline Tardíos & 0,308 & $-0,164$ \\
\hline Constante & $-0,251$ & $-0,618$ \\
\hline$-2 L L$ & 1019,468 & 996,338 \\
\hline
\end{tabular}

${ }^{*} p<0,100 ;{ }^{* *} p<0,050 ;{ }^{* * *} p<0,010$

Fuente: ENHALI-2012.

\section{¿HAY ANOMIA ALIMENTARIA?}

Otro aspecto fundamental de este artículo es determinar si en nuestra sociedad la alimentación se ha convertido en un hábito anómico, cuyas normas sobre el comer se han individualizado, o si, por el contrario, se siguen manteniendo reglas compartidas de elección de los alimentos. Una forma de aproximarnos a esta cuestión es analizar los criterios que emplean las personas que cocinan para componer los menús diarios. Se han establecido tres grupos de criterios. Un primer grupo está representado por 
los gustos, tanto de la persona que cocina como de los otros comensales. En segundo lugar figuran los criterios relacionados con la salud: la dieta y el equilibrio y/o composición de los platos. Por último, se ha establecido un tercer grupo integrado por criterios que podríamos considerar más racionales: el precio de la comida, el número de comensales y la facilidad de preparación.

Antes de comentar los resultados obtenidos, cabe señalar que, en concordancia con las desigualdades de género existentes en España en el reparto de las tareas domésticas (Encuesta de Empleo del Tiempo 2010), un $77 \%$ de las personas que son responsables de todo o de la mayoría de lo que se cocina en casa son mujeres, frente a un $23 \%$ de hombres. Una vez efectuada esta matización, los criterios de elección de los alimentos por parte de las personas que cocinan denotan una preocupación importante por lo que se come y unos conocimientos sobre lo adecuado e inapropiado nada desdeñables, pero también una predisposición relevante hacia la consideración de los gustos como factor fundamental de decisión para cocinar. Así, los aspectos ligados a la salud y el equilibrio de los platos están entre las prioridades de elección de alimentos para quien cocina, pues un $51 \%$ afirma seleccionar por salud y también un $27 \%$ dice hacerlo por equilibrio y composición. Pero también el criterio del gusto, el propio o el del resto de los comensales, es prioritario. El gusto domina las selecciones, pues un $55 \%$ de las personas que cocinan adoptan este criterio para elaborar la comida diaria (Cuadro 5).

El precio es un criterio destacado, aunque secundario, señalado por el $22 \%$ de los entrevistados que cocina. Esto no está indicando que el precio sea irrelevante; puede deberse solamente al hecho de que es posible priorizar otros criterios sin aumentar los costes, o que incluso es posible reducir los costes sin que se altere el ideal de dieta saludable y placentera que marca la cultura alimentaria nacional. Este aspecto está directamente ligado a la oferta alimentaria.

Es importante determinar si los criterios de elección de los alimentos son similares entre los distintos grupos sociales o si, por el contrario, las diferencias son acusadas. Para ello se ha analizado la relación de los criterios que se siguen en la elección de los alimentos con las principales variables de estratificación social: sexo, edad, estudios, situación laboral y tamaño del lugar de residencia.

De los resultados expuestos en el Cuadro 5 se deduce que existe una elevada uniformidad sobre los criterios de elección de los alimentos: la salud y el placer parecen dominar las elecciones, independientemente del perfil del cocinero o cocinera. Esto indica una importante estabilidad en las creencias y los valores ligados a la alimentación.
No obstante, estos resultados no quieren decir que no existan diferencias significativas entre los distintos grupos sociales, sobre todo en el orden de prioridad de algunos criterios. Para determinar si existe asociación entre los criterios que intervienen en la elección de alimentos y las variables sociodemográficas consideradas, se han realizado pruebas de significación para preguntas múltiples. Como puede observarse en el Cuadro 6, todas las variables sociodemográficas presentan una asociación estadísticamente significativa.

Así, aunque se coincida en los criterios principales, los datos del Cuadro 5 indican que las mujeres dan más importancia a la salud que los hombres; y a éstos les preocupa en mayor medida la facilidad de preparación de los platos. Según la edad, a los jóvenes también les guía más en sus elecciones la facilidad de preparación de los alimentos respecto al resto de grupos de edad, mientras que la salud está más presente en las personas mayores. Respecto a la variable estudios, cuanto mayor es el nivel educativo menor es la importancia otorgada a los gustos propios o del resto de comensales y más interesado se está en el equilibrio y/o composición de los platos. Si se considera la situación laboral de las personas que cocinan, los estudiantes están menos interesados en el equilibrio y/o composición de los platos y en la dieta, mientras que destacan por su preocupación por la facilidad de preparación de las comidas. Los jubilados priorizan más la salud que los gustos propios o de otros comensales y, curiosamente, constituyen el grupo al que menos le importa el precio de los alimentos; un criterio que es más valorado por los parados. Por último, si se considera el tamaño del hábitat, son los individuos que viven en zonas con un menor número de habitantes los que más tienen en cuenta la salud. No hay que olvidar que los pequeños municipios suelen caracterizarse por el alto grado de envejecimiento de la población. Asimismo, el criterio de equilibrio y/o composición de los platos está más presente en las personas que residen en las grandes ciudades, aunque también es elevado en los municipios de 10.001 a 100.000 habitantes.

\section{Conclusiones}

La sociedad española no muestra una alimentación desestructurada, pero sí algunos rasgos de interés para comprender sus particularidades. Se observa una manifiesta sincronía horaria, pues toda la población resuelve su alimentación con dos comidas principales, lo cual representa una continuidad con los hábitos tradicionales: ni la alimentación diaria se apoya en una sola comida, ni se resuelve con múltiples ingestas secundarias, ni se realiza mayoritariamente fuera del hogar. Los horarios, sin embargo, sí ofrecen pautas de transformación, pues las franjas de las ingestas se amplían, especialmente en el tardío horario de las cenas. 
Cuadro 5.

Criterios de elección de alimentos según sexo, edad, nivel de estudios y situación laboral (Respuesta múltiple)

\begin{tabular}{|c|c|c|c|c|c|c|c|}
\hline & Gustos & Salud & Precio & Facilidad & Dieta & $\begin{array}{l}\text { Número de } \\
\text { comensales }\end{array}$ & Equilibrio \\
\hline Total & 54,7 & 51,0 & 22,1 & 7,9 & 14,1 & 1,5 & 26,7 \\
\hline \multicolumn{8}{|l|}{ Sexo } \\
\hline Varón & 59,4 & 43,4 & 19,4 & 12,6 & 12,6 & 0,6 & 28,0 \\
\hline Mujer & 55,0 & 54,2 & 23,4 & 6,9 & 14,8 & 1,8 & 27,1 \\
\hline \multicolumn{8}{|l|}{ Edad } \\
\hline $18-30$ & 48,3 & 46,0 & 23,0 & 13,8 & 12,6 & 1,1 & 29,9 \\
\hline $31-40$ & 57,1 & 52,1 & 23,5 & 15,1 & 16,0 & -- & 24,4 \\
\hline $41-55$ & 59,1 & 52,6 & 24,4 & 7,9 & 16,8 & 1,4 & 30,9 \\
\hline $56-65$ & 61,5 & 51,9 & 23,5 & 6,4 & 9,1 & 1,6 & 30,5 \\
\hline $66-75$ & 48,4 & 57,8 & 18,8 & 3,1 & 16,4 & 3,9 & 21,9 \\
\hline+75 & 50,0 & 48,8 & 18,6 & 3,5 & 14,0 & 1,2 & 17,4 \\
\hline \multicolumn{8}{|l|}{ Estudios } \\
\hline Menos que primarios & 61,4 & 50,5 & 25,7 & 4,0 & 9,9 & 3,0 & 19,8 \\
\hline EGB, ESO & 61,4 & 55,0 & 23,9 & 5,6 & 13,1 & 2,8 & 23,1 \\
\hline Bachiller, BUP, COU & 61,8 & 47,1 & 19,7 & 9,6 & 18,5 & 0,6 & 21,7 \\
\hline FP & 51,4 & 51,4 & 24,3 & 6,5 & 16,8 & -- & 29,0 \\
\hline U. medios & 48,7 & 52,1 & 22,2 & 9,4 & 17,9 & 0,9 & 41,0 \\
\hline U. superiores & 46,7 & 53,9 & 20,6 & 12,7 & 10,9 & 1,2 & 32,7 \\
\hline \multicolumn{8}{|l|}{ Situación laboral } \\
\hline Trabaja & 57,5 & 48,1 & 20,1 & 11,3 & 14,2 & 0,9 & 31,8 \\
\hline Parado & 57,0 & 57,0 & 29,0 & 8,0 & 17,0 & 2,0 & 30,0 \\
\hline Estudiante & 51,6 & 45,2 & 25,8 & 29,0 & 9,7 & -- & 16,1 \\
\hline Jubilado & 47,4 & 54,7 & 19,8 & 4,2 & 13,0 & 2,1 & 24,5 \\
\hline Ama de casa & 60,3 & 54,1 & 24,9 & 4,3 & 15,2 & 1,9 & 24,1 \\
\hline \multicolumn{8}{|l|}{ Tamaño hábitat } \\
\hline-10.000 & 57,4 & 62,8 & 24,8 & 6,2 & 19,4 & 3,9 & 21,7 \\
\hline $10.001-100.000$ & 57,5 & 52,5 & 25,1 & 7,1 & 14,0 & 1,3 & 34,0 \\
\hline $100.001-500.000$ & 55,4 & 50,7 & 19,1 & 10,4 & 11,9 & 1,1 & 20,1 \\
\hline+500.000 & 50,0 & 42,0 & 20,5 & 7,1 & 16,1 & 0,9 & 28,6 \\
\hline
\end{tabular}

Fuente: ENHALI-2012.

\section{Cuadro 6.}

Pruebas de independencia (Chi-cuadrado*)

\begin{tabular}{|c|c|c|c|}
\hline & Chi-cuadrado & GI & Significación \\
\hline Sexo & 22,729 & 8 & 0,004 \\
\hline Edad & 62,107 & 40 & 0,014 \\
\hline Estudios & 72,054 & 40 & 0,001 \\
\hline Situación laboral & 63,581 & 32 & 0,001 \\
\hline Tamaño de hábitat & 55,250 & 24 & 0,000 \\
\hline Tener hijos de más de 15 años & 31,181 & 8 & 0,000 \\
\hline
\end{tabular}

* El estadístico de chi-cuadrado es significativo en el nivel 0,05.

Fuente: ENHALI-2012.

En los horarios de la comida del mediodía son los trabajadores los responsables de la ampliación de la franja horaria. Por el contrario, son las personas no insertas en el mercado de trabajo las que comen más temprano. Las cenas tienen dos picos hora- rios, uno en torno a las nueve y otro alrededor de las diez; y aunque no hay un perfil claro de quiénes cenan temprano, el factor que más afecta al horario de la cena, obligando a una ingesta tardía, es también el empleo, junto con los estudios. En esen- 
cia, el alargamiento horario se produce por motivos ajenos al hogar (por trabajo y estudios), pero cabe también pensar que no se trata solamente de una imposición externa, sino de una adaptación de estas constricciones a los deseos del grupo por coincidir a la hora de comer o cenar. Los grupos que no tienen que esperar a que otros miembros del hogar lleguen a casa (los jubilados), tienen comidas más tempranas. En los hogares con ocupados el período de la cena se dilata, por lo que cabe imaginar a una mujer que espera por sus hijos estudiantes o a su marido, alargando el horario para buscar la coincidencia familiar. En definitiva, estamos ante un rasgo de dispersión horaria que se realiza para buscar coincidencias con el grupo, más que ante una manifestación de la desestructuración del ritmo de comidas.

En relación con el papel de la familia en la alimentación destaca el componente relacional en todas las comidas realizadas a lo largo del día; se come y se cena principalmente en el hogar y en familia, siendo las comidas secundarias las más solitarias. No parece que la sociabilidad se vea afectada, ya que no destaca la comida en solitario, pues comen solos los que viven solos; pero incluso entre este colectivo es frecuente comer en compañía. Se está visibilizando el carácter familista de la sociedad española, pues las personas que viven solas acuden a casa de sus familiares a comer o cenar: padres mayores que van a comer al hogar de sus hijos o hijos que mantienen la vinculación con la familia de origen a través de la comida. Se trata de personas que cambian de hogar para resolver su alimentación cotidiana y que, en lugar de hacerlo en servicios de restauración o con amigos, optan por el hogar de la familia para evitar comer solos y/o para mantener el vínculo familiar. Cabe señalar, no obstante, que una de cada cuatro personas que viven acompañadas come y cena sola; y que hay datos para pensar en otros tipos de sociabilidad que vinculan la alimentación a las relaciones no estrictamente familiares, como las comidas con compañeros de trabajo o con amigos. Sin duda, esto requeriría un estudio específico. Convendría también indagar en el perfil de la persona sola, pues los datos apuntados por el IMSERSO (2015) revelan que, entre las personas mayores que viven solas, existe el triple de mujeres que de varones. Este dato reforzaría aún más el vínculo con las familias de origen, dadas las mayores destrezas culinarias de las mujeres de la generación de mayores. No obstante, no contamos con datos para confirmar si estos movimientos entre hogares están relacionados o no con la edad de quien vive en soledad.

En relación con la anomia alimentaria en la elección de los alimentos, los datos muestran que, en España, las personas que cocinan emplean cri- terios consistentes para la confección de las dietas diarias y que existen reglas de conducta reconocibles: el equilibrio de los platos y la salud son normas compartidas para decidir qué comer. Pero también los criterios relativos al gusto de los comensales les unifican, mostrándose de nuevo el componente relacional de la comida que hace que las personas encargadas de esta tarea no pierdan de vista a los comensales a la hora de preparar los platos diarios. Cabe suponer que las decisiones están mediadas por la oferta alimentaria de cada país $y$, en este caso, todo indica que los españoles han podido reducir sus gastos alimentarios durante la crisis económica sin alterar sustancialmente la dieta, pues el factor precio no destaca entre la mayoría de los entrevistados.

Se puede afirmar que se comparten criterios sobre lo apropiado e inapropiado. Sin embargo, la fuerte división de género en la preparación de la comida hace pensar que estos criterios son más propios de las mujeres responsables de la comida doméstica, no tanto un conjunto de normas generalizadas entre la población. Así, los hombres son menos proclives a elecciones saludables y a los jóvenes les preocupa la facilidad para cocinar, pero cuando hay un responsable de la alimentación del grupo los criterios se homogeneizan. No obstante, la falta de datos, tanto en este estudio como en otras encuestas, acerca de cómo afecta el criterio del gusto en la distribución de los alimentos dentro del hogar, no permite confirmar que el criterio de salud sea homogéneo en la distribución de los alimentos. Puede suceder que las dietas sean diversas, bien por necesidades especiales de los comensales o por el interés de quien elabora los alimentos en respetar las peculiaridades del grupo. Esto supondría una heterogeneidad en la alimentación domestica que sería interesante explorar con un estudio específico y que marcaría la homogeneidad aquí reflejada.

Los resultados descritos en este artículo muestran algunos rasgos propios de la alimentación española que conviene resaltar.

En primer lugar, es preciso considerar que el entorno de sociabilidad que propicia el fuerte vínculo familiar de la sociedad española se detecta con claridad en el hábito de comer en compañía y en el hogar familiar. Compartir la comida es más fácil en un contexto en el que existe este elemento cultural que vincula a los individuos entre sí y que encuentra en la comida un punto de encuentro para reforzar y recrear los lazos familiares, sean estos en el mismo hogar o fuera de él.

Un segundo aspecto a tener en cuenta es la centralidad de la alimentación en la vida diaria, reforzada o validada por dos cuestiones: por un lado, por el hecho de contar con una jornada laboral partida, que divide el día de trabajo en dos partes; 
pero, sobre todo, por contar con dos comidas principales (la comida y la cena), y no solo una, como en otros contextos alimentarios. La jornada laboral y escolar se detiene para comer, igual que se detiene al final del día. Así, la hora de la comida y de la cena constituyen los dos momentos de encuentro, en este caso familiar y en el hogar.

El tercer elemento que explica la concordancia de hábitos alimentarios de una sociedad como la española, tan diversa en otros aspectos, es el rol de la persona responsable de la alimentación del hogar. Por una parte, quien se ocupa de la alimentación exime al resto de esta responsabilidad, asume la tarea de la gestión de la comida y la compra y hace atractivo el hogar como lugar de consumo, por la facilidad de contar con la comida preparada y por el gran ahorro que supone comprar con cargo a un mismo presupuesto. Aunque esto también es una vía de ahorro para quien vive en soledad y es responsable de su propia alimentación, no es posible ignorar que tres de cada cuatro personas responsables de la alimentación del hogar son mujeres.

Por ello, el cuarto elemento a tener en cuenta para explicar los hábitos alimentarios de los españoles es el género, concretamente el papel predominante de las mujeres en el rol de cuidadoras de la alimentación del hogar. En este rol y en esta desigualdad de género está implícita la homogeneidad de la norma alimentaria, pues ellas deciden por otros lo que es bueno y malo comer y lo ponen en práctica. Con esta práctica las mujeres reproducen las normas alimentarias y los demás las aceptan, las asumen y también las aprenden. Pero, además, ellas son las principales responsables de gestionar los horarios y hacer coincidir al grupo, de preparar y escoger la comida pensando en los gustos de los comensales y en su salud y de reunir al grupo en torno a la mesa. A través de la gestión de la alimentación se asegura el encuentro y la sociabilidad del grupo, también la reproducción de las normas alimentarias.

Los datos aquí presentados, por tanto, no reflejan una absoluta homogeneidad, pero tampoco una alimentación desestructurada ni en normas ni en horarios; ni muestran tal heterogeneidad en los hábitos que nos obligue a pensar en una alimentación con creciente individualización o desregulada. La alimentación se estructura en torno a la familia, el motivador principal para su reproducción es la sociabilidad y el eje sobre el que se enlazan estos dos elementos es la existencia de una persona que cocina y compra para otros. La unión en torno a la comida ofrece un escenario de orden frente a la heterogeneidad de la vida cotidiana de cada uno de los miembros del grupo, frente a la diversidad de trayectorias individuales, haciendo que las im- posiciones externas no generen desestructuración. Comer podría ser algo individual y diverso, aunque aquí se resuelve de manera colectiva y en el ámbito familiar. Pero esto también refleja una gran debilidad, pues una buena parte de esta estabilidad alimentaria se sustenta en el rol tradicional de género de las mujeres en el hogar como cocineras y gestoras de la alimentación cotidiana.

Quedan abiertas algunas líneas de trabajo interesantes para seguir analizando la alimentación española y sus cambios que ayuden a completar las hipótesis aquí planteadas.

En primer lugar, no se puede analizar hoy la alimentación sin la presencia de agentes de socialización complementarios de la familia. El efecto que la socialización no familiar puede producir al modificar los hábitos de consumo, en especial en la población más joven, resulta complementaria a la producida en el hogar y debería ser analizada para ver el peso de la socialización familiar en los gustos y los hábitos.

En segundo lugar, recuperamos la idea de la posible heterogeneidad interna de la familia en sus hábitos culinarios. Si los gustos son una prioridad para quien cocina, bien puede suceder que se adapten las dietas a ellos y que los miembros de la familia coman juntos aunque comidas diferentes. Si añadimos a esto las dietas o peculiaridades ligadas a la salud que puedan exigir los comensales, deberíamos considerar la posibilidad de una exploración interna del hogar que permitiera detectar la heterogeneidad que queda oculta en un estudio cuantitativo general, como el que aquí se plantea. Como es habitual en todos los análisis de conductas complejas, como las alimentarias, se requieren aproximaciones cualitativas que completen los vacíos de los estudios cuantitativos.

En último lugar, quizás por la relevancia que tiene la comida para vertebrar al grupo, no debamos preguntarnos solamente cuáles son los factores que propician su desestructuración o su estabilidad, sino profundizar en el papel central de la alimentación como generadora de pautas de conducta que oponen resistencia a la desestructuración impuesta por los procesos de modernización de la vida social. No estamos ante una fuerza única que mueva los hábitos alimentarios hacia la individualización, sino ante comportamientos colectivos que atan a los individuos entre sí. No hay solo fuerzas de desestructuración o descenso de las disciplinas alimentarias, sino también normas alimentarias firmes y transmitidas a través de la elección de alimentos. El modelo de Warde (1997), basado en la confluencia de fuerzas, está funcionando, tomando aquí protagonismo lo comunitario y lo grupal. 


\section{RefERENCIAS Bibliográficas}

Atkinson, W. y C. Deeming. 2015. "Class and cuisine in contemporary Britain: the social space, the space of food and their homology". The Sociological Review 63 (4): 876-896. https://doi.org/10.1111/1467-954X.12335

Bourdieu, P. 1998. La distinción: Criterio y bases sociales del gusto. Madrid: Taurus.

Charles, N. y M. Kerr. 1986. "Issues of responsibility and control in the feeding of families". En The Politics of health education: raising the issues, editado por S. Rodmell y A. Watt. London: Routledge.

Cheng, S-L., W. Olsen, D. Southerton y A. Warde. 2007. "The changing practice of eating: evidence from UK time diaries, 1975 and 2000". British Journal of Sociology 58(1): 39-61. https://doi.org/10.1111/j.14684446.2007.00138.x

Contreras, J. 2002. "La modernidat alimentaria: entre la desestructuracio i la proliferació de codis". Pp. 349-363 en La vida quotidiana a traves dels segles. Barcelona: Pórtic.

Contreras, J. y M. Gracia Arnaiz. 2004. Observatorio de la alimentación. Barcelona: Alimentaria.

Davidson R y A.H. Gauthier. 2010. "A cross-national multilevel study of family meals". International Journal of Comparative Sociology 51(5): 349-365. https://doi. org/10.1177/0020715210376853

Díaz Méndez y C. Gómez Benito. 2005. "Consumo, seguridad alimentaria y salud: una perspectiva de análisis desde las Ciencias Sociales". Revista Internacional de Sociología. 40: 9-19. https://doi.org/10.3989/ris.2005. $\mathrm{i} 40.187$

Díaz Méndez, C. y García Espejo. 2014. "Eating practice models in Spain and the United Kingdom: A comparative time-use analysis". International Journal of Comparative Sociology 55(1): 24-44. https://doi. org/10.1177/0020715213519657

Díaz Méndez, C. y J. Callejo. 2014. "Homogenization of eating times in the United Kingdom and Spain". British Food Journal 116(5): 734-752. https://doi.org/10.1108/BFJ05-2012-0121

ENHALI. 2012. Encuesta de hábitos alimentarios de los españoles. Madrid. Ministerio de Agricultura, Alimentación y Medio Ambiente.

Eurobarometer 2010. Special Eurobarometer 354: Foodrelated risks. Bruselas: European Commission.

Ficshler, C. 1979. "Gastro-nomie et gastro-anomie". Communications 31(1): 189-210. https://doi. org/10.3406/comm.1979.1477

Gallegos, D., S. Dziurawiec, F. Fozdar y L. Abernethie. 2011. "Adolescent experiences of 'family meals' in Australia". Journal of Sociology 47: 234-260. https:// doi.org/10.1177/1440783310386826

González Turmo, I. 1995. Comida de rico, comida de pobre: los hábitos alimenticios en el occidente andaluz. Sevilla: Servicios de publicaciones de la Universidad de Sevilla.

Gracia Arnaiz, M.I. 1997. La transformación de la cultura alimentaria. Cambios y permanencias en un contexto urbano. Madrid: Ministerio de Educación y Cultura.

Gracia Arnaiz, M.I. 2003. "Alimentación y cultura: Hacia un nuevo orden alimentario". Pp. 205-230 en Los modelos alimentarios a debate: Interdisciplinariedad de la alimentación. Murcia: Universidad Católica San Antonio.

Gracia Arnaiz, M.I. 2008 "Nuevas maneras de vivir, nuevas maneras de comer. Comer hoy en España". Distribución y Consumo 17: 5-18.

Grignon, C. y Ch. Grignon. 2004. "Sociologie des rythmes alimentaires". Pp. 245-257 en Les comportements alimentaires. Paris: Lavoisier.

Grignon, C. y Ch. Grignon. 1981. "Alimentation et stratification social". Cahiers de nutrition et diététique 16(4): 208-240.

Grignon, C. y Ch. Grignon. 1980. "Styles d'alimentation et gouts populaires". Revue française de Sociologie XXI: 531-569. https://doi.org/10.2307/3320833

Herpin, N. 1998. "Les repas comme institution, compte rendu d' une enquête exploratoire". Revue Française de Sociologie 29: 503-552. https://doi. org $/ 10.2307 / 3321627$

Holm, L., U. Kjaernes y D.S. Lauridsen. 2014. "Lunchinstitutional framing of changes in everyday eating-a comparative explotarion". Paper presentado en ESA Consumption Conference, Porto 2014.

IMSERSO 2015. Informe 2014. Las personas mayores en España. Datos estadísticos estatales y por comunidades autónomas. Madrid: Ministerio de Sanidad, Servicios Sociales e Igualdad.

Instituto Nacional de Estadística. 2010. Encuesta de Empleo del Tiempo. Madrid: INE.

Instituto Nacional de Estadística. 2011. Censo de población y vivienda. Madrid: INE.

Kemmer, A., S. Anderson y D.W. Marshall. 1998. "Living together and eating together: changes in food choice and eating habits during the transition from single to married/cohabiting". The Sociological Review 46 (1): 48-72. https://doi.org/10.1111/1467-954X.00089

Lambert, J. L. 1987. L'évolution des modèles de consommation alimentaire en France. Paris: Technique et Documentation-Lavoisier.

Lhuissier, A., C. Tichit, F. Caillavet, P. Cardon, A. Masullo, J. Martín-Fernández, I. Parizot y P. Chauvin. 2013. "Who still eats three meals a day? Findings from a quantitative survey in the Paris area". Appetite 63: 5969. https://doi.org/10.1016/j.appet.2012.12.012

Lupton, D. 1996. Food, culture and the self. London: Sage Publication.

Marshall, D.W. y A.S. Anderson. 2002. "Proper meals in transition: young married couples on the nature of eating together". Appetite 39(3): 193-206. https://doi. org/10.1006/appe.2002.0507

Martín-Lagos López, M.D. 2011. "Consumption and Modernization in the European Union". European Sociological Review 27: 124-137. https://doi. org/10.1093/esr/jcq001

Medina, X. 2005. Food culture in Spain. Westport: Greenwood Publishing Group.

Mestdag I. e I. Glorieux. 2009. "Change and stability in commensality patterns: a comparative analysis of Belgian time-use data from 1966, 1999 and 2004". Sociological Review 57: 703-726. https://doi. org/10.1111/j.1467-954X.2009.01868.x 
Mestdag, I. y J. Vandeweyer. 2005. "Where has family time gone? in search of joint family activities and the role of the family meal in 1966 and 1999". Journal of Family History. 30: 304-323. https://doi. org/10.1177/0363199005275794

Multinational Time Use Study 2011. Centre for Time Use Research. Oxford: University of Oxford. www.timeuse. org/mtus [Consulta 8 de mayo de 2011].

Murcott, A. 1982. "On the social significance of the 'cooked dinner' in South Wales". Social Science Information 21: 677-696.

OCHA 1993. Les Français et I'harmonie alimentaire. Cahiers de I'Ocha. http://www.lemangeur-ocha.com/ouvrage/ les-francais-et-Iharmonie-alimentaire/ [Consulta 10 de enero de 2011].

Poulain, J. P. 2002. "The contemporary diet in France: 'destructuration' or from commensalism to 'vagabond feeding'”. Appetite 39: 43-55. https://doi.org/10.1006/ appe.2001.0461

Poulain, J. P. 2008. Manger Aujourd'hui. Cahiers de l'Ocha. http://www.lemangeur-ocha.com/ouvrage/mangeraujourdhui/ [Consulta 10 de enero de 2011].

Serra Majem, L., L. Ribas Barba, B. Roman Viñas y J. Aranceta Bartrina. 2003. "Hábitos alimentarios y consumo de alimentos en la población infantil y juvenil española (1998-2000): variables socioeconómicas y geográficas". Medicina Clínica 121(4): 126-131.

Sobal, J., C.F. Bove y B.S. Rauschenbach. 2002. "Commensal careers at entry into marriage: establishing commensal units and managing commensal circles". The Sociological Review 50 (3): 378-397. https://doi. org/10.1111/1467-954X.00388

Sorokin, P.A. y R.K. Merton. 1937. "Social Time: a methodological and functional analysis". American Journal of Sociology. XLII(5): 615-629. https://doi. org/10.1086/217540

Warde, A. 1997. Consumption, Food and Taste. London: Sage.

Warde, A., S. Cheng, W. Olsen y D. Southerton. 2007. "Changes in the practice of eating: a comparative analysis of time-use". Acta Sociológica 50(4): 363-385. https://doi.org/10.1177/0001699307083978

Zerubavel, E. 1981. Hidden Rhythms: Schedules and Calendars in Social Life. Chicago: University of Chicago Press.

CECILIA DÍAZ MÉNDEZ es catedrática acreditada de Sociología en la Universidad de Oviedo. Entre sus trabajos se incluyen "Nutrition and the Mediterranean diet. A historical and sociological analysis of the concept of "health diet" in Spanish society". Food Policy (Díaz-Méndez y Gómez Benito, 2010); "Behaviour change and the temporal ordering of eating practices: a UK-Spain comparison". International Journal of Sociology of Agriculture and Food (Southerton, Díaz-Méndez y Warde, 2012) y "Eating Practice Models in Spain and the United Kingdom: a Comparative Time Use Analysis". IJCS (Díaz-Méndez y Garcia-Espejo, 2014). Es Directora del Grupo de Investigación de Sociología de la Alimentación.

ISABEL GARCÍA ESPEJO es profesora titular de Sociología en la Universidad de Oviedo. Entre sus trabajos se incluyen "Educational-Skill Matches and Labor Achievements among Graduates in Spain". European Sociological Review, (García-Espejo e Ibáñez, 2006); "Eating Practice Models in Spain and the United Kingdom: a Comparative Time Use Analysis". International Journal of Comparative Sociology, (Díaz-Méndez y Garcia-Espejo, 2014), y "Tendencias en la alimentación contemporánea: la homogeneización del gasto alimentario en España y Reino Unido". Revista Española de Investigaciones Sociológicas (Díaz Méndez y García Espejo, 2012), "Eating out in Spain: Motivations, sociability and consumer context”, Appetite, (Díaz-Méndez and Garcia -Espejo, 2017). 